\title{
KDM4D Predicts Recurrence in Exocrine Pancreatic Cells of Resection Margins from Patients with Pancreatic Adenocarcinoma
}

\author{
JOEL ISOHOOKANA $^{1}$, KIRSI-MARIA HAAPASAARI ${ }^{2}$, YLERMI SOINI $^{2,3}$ and PEETER KARIHTALA ${ }^{1}$ \\ ${ }^{1}$ Department of Oncology and Radiotherapy, Medical Research Center Oulu, \\ Oulu University Hospital and University of Oulu, Oulu, Finland; \\ ${ }^{2}$ Department of Pathology, Medical Research Center Oulu, \\ Oulu University Hospital and University of Oulu, Oulu, Finland; \\ ${ }^{3}$ Department of Pathology and Forensic Medicine, Cancer Center of Eastern Finland, \\ University of Eastern Finland, Kuopio, Finland
}

\begin{abstract}
Background/Aim: The role of histone demethylators, such as Jumonji domain 2 (JMJD2/KDM4) proteins, and histone deacetylases, such as sirtuins (SIRT) is poorly characterized in pancreatic carcinomas while they have a major role in the carcinogenesis of several other tumours. Materials and Methods: We assessed retrospectively with immunohistochemistry the expressions of KDM4A, KDM4B and KDM4D in 81 and SIRT1-4 in 102 pancreatic adenocarcinomas. Immunostaining was evaluated separately in benign pancreatic tissues and in malignant cells. Results: High nuclear KDM4D expression in benign pancreatic tissue from resection margins associated with dismal disease-free survival (DFS) $(O R=8.00 ; \quad 95 \% C I=1.87-33.9 ; \quad p=0.005)$, even more significantly than tumour size and lymph node involvement. High cytoplasmic SIRT2 expression in benign pancreatic tissues also associated with a shorter DFS, but only in univariate analysis $(p=0.026)$. Conclusion: Nuclear KDM4D and SIRT2 expression deviated from that of benign pancreatic tissue thus putatively influencing gene expression of tumor cells. Regardless, none of the enzymes studied had a decisive role in the spread of pancreatic cancer. A high nuclear expression of KDM4D in samples of pancreatic resection margins significantly and independently predicted an earlier recurrence and could thus be used in the assessment of risk of relapse in clinical practice.
\end{abstract}

Correspondence to: Peeter Karihtala, Department of Oncology and Radiotherapy, Oulu University Hospital, PO Box 22, 90029 Oulu, Finland. Tel: +35883152011, e-mail: peeter.karihtala@oulu.fi

Key Words: Epigenetics, immunohistochemistry, KDM4, sirtuin, pancreatic cancer.
Within the next decade pancreatic cancer is predicted to be the second leading cause of cancer-related death in the Western societies while the prognosis remains poor and the 5-year survival rate is $<5 \%$ (1). Mainly due to tumour involving local vessels, curative surgical treatment is achievable only in 15$20 \%$ of cases. The progression of therapies, including adjuvant treatments, still have only a slight improvement for overall survival (2). Better knowledge of heterogenic pancreatic cancer tumour biology and tumour subtyping could provide advanced prognostic and predictive information for different subtypes of the disease (3). Although novel prognostic and predictive factors are under extensive search in pancreatic cancer, TNMclassification and CA19-9 value are still the only factors, that can guide clinical decision making (4-7).

Chromatin in mammalian cells consists of genomic DNA, histones, regulatory proteins and non-coding RNAs (8). Nucleosome is a fundamental unit of chromatin where 1.47 rounds of negatively charged DNA is wrapped around a positively charged histone, an octamer which is built from core histone proteins: $\mathrm{H} 2 \mathrm{~A}, \mathrm{H} 2 \mathrm{~B}, \mathrm{H} 3$, and $\mathrm{H} 4$. In addition, $\mathrm{H} 1$ is a linker histone between nucleosomes $(8,9)$. Epigenetics imply the changes in gene expression that are not coded in the DNA sequence itself (10). The main epigenetic modifications in the normal development and growth of cells are DNA methylation, histone modifications and post-transcriptional gene regulation by microRNAs (11). These modifications jointly influence the regulation of genome function by modifying the regional structure dynamics of chromatin, primarily regulating its accessibility and compactness (12).

Reversible histone acetylation and methylation are the two most predominant post-translational modifications of histone tails that regulate gene expression $(8,11)$. The Jumonji $\mathrm{C}$ Domain (JMJD) family consists of 30 proteins in mammals, 18 of them having demethylating enzyme activity of histone 
residues. JMJD proteins are classified in subfamilies according to the degree of homology and the presence of other domains. JMJD2A-D, also known as lysine (K)-specific demethylase 4 (KDM4), is one of the JMJD subfamilies in humans, where JMJD2/KDMA-C are $120 \mathrm{kDa}$ proteins and present double PHD and Tudor domains, which are implicated in binding to methylated histones. JMJD2D/KDM4D is only half the size of other KDM4 members and has no PHD and Tudor domains. KDM4A and KDM4B have as a substrate H3K9 and H3K36 and KDM4D H3K9 and H1.4K26. By mono-, di- or tridemethylating lysine residues the JMJD/KDM4 proteins are involved in various processes within the cell, including regulation of gene transcription, epigenetic silencing, heterochromatin formation, genomic imprinting and DNA repair. Dysregulation of JMJD protein activity is related to progression of cancer $(9,13,14)$. Highly selective KDM inhibitors have been recently characterized (15).

Histone deacetylases (HDACs) are categorized into four classes based on homology to yeast HDACs (8). Class III HDACs consist of seven isoforms of the silent information regulatory 2 (Sir2) enzyme family called sirtuins (SIRTs), which function in the nucleus, cytoplasm and mitochondria (16). Sirtuins are NAD dependent histone deacetylases and/or mono (ADP-ribosyl) transferases which regulate genome stability, cellular metabolism, and lifespan. As a prominent function of HDACs, sirtuins intercedes epigenetic silencing by deacetylase modification of lysine residues of histones but also various non-histone substrates (16-18).

Mammalian sirtuins are classified into four different subclasses: SIRT 1-3 belongs to class I, SIRT4 to class II, SIRT5 to class III and SIRT 6-7 to class IV (19). Class I members have strong deacetylase activity in vitro and class II member (SIRT4) acts as a mono-ribosyltransferase and has a weak deacetylase activity in vitro $(19,20)$. SIRT1 functions primarily as a nuclear deacetylase. SIRT1 removes the acetyl group from the $\varepsilon$-amino group of lysine residues in histones (H4K16 and H3K9) and non-histone proteins, and regulates target gene expression and protein activities that control multiple cellular processes such as cell proliferation, differentiation and apoptosis. Several functions of SIRT1 depend on its ability to deacetylate histone H3K9 and H4K16 and to mediate consecutive transcriptional repression $(19,21)$.

SIRT2 is mainly localized in the cytoplasm but it has also the ability to transfer to the nucleus during mitosis. SIRT2 deacetylates histone substrates H4K16, H3K56 andH3K9 but also various of non-histone substrates. p53 is perhaps the best known non-histone substrate of SIRT1 and SIRT2. SIRT2 regulates several cell functions including cell cycle progression, cell death, and stress response $(17,20,22,23)$. SIRT3 and 4 localize mainly in the mitochondria, where they regulate diverse mitochondrial proteins. SIRT3-4 function mainly in metabolic regulation. However, recent reports suggested that SIRT3 plays a role in epigenetic regulation through deacetylation of histone H4K16 and H3K9, although it remains controversial $(19,20,22)$.

In this study we collected material from surgically treated pancreatic adenocarcinomas in order to evaluate the mainly unknown role of KDM4 and SIRT families as potential prognostic factors of this disease. Emphasize was given to characterize expressions separately in benign pancreatic cells taken from resection margins and malignant tumor tissue.

\section{Materials and Methods}

Samples. The material consisted of a retrospective cohort of pretreatment surgical pancreatic adenocarcinoma samples from 102 patients in SIRT1-4 stainings and 81 patients in KDM4A, KDM4B and KDM4D stainings. The number of immunostanings between KDM4 and SIRT varied due to the lack of reliably representative material for KDM4 stainings in 21 cases. Overall, 97 (95.1\%) of the patients underwent pancreaticoduodenectomy. To assess the value of the studied markers to predict recurrence, samples were assessed both from malignant adenocarcinoma cells and also separately from benign exocrine pancreatic tissues from resection margins, when available. The specimens had been fixed in neutral formalin, embedded in paraffin blocks and stored at the Department of Pathology in Oulu. All patients were diagnosed and treated in Oulu University Hospital, Oulu, Finland during 1993-2015. 50 $(49.0 \%)$ of the patients were diagnosed in 2010 or after that. During the follow-up time (median 15 months) 72 patients (70.6\%) died of pancreatic cancer. Diagnoses were reviewed by a specialist pathologist and the evaluation of immunostaining was performed by experienced histopathologists $(\mathrm{KMH})$ and (JI). Exact and updated patient data were acquired from patient medical records. During the evaluation of the immunostainings, the clinical patient data were blinded. Pathological TNM staging was available in $99(97.1 \%)$ cases and only clinical TNM staging was available in $2(2.0 \%)$ cases. In one of the cases, a reliable TNM staging was absent.

Immunohistochemistry. Pancreatic adenocarcinoma samples and samples from benign pancreatic tissue from resection margins were fixed in formalin and embedded in paraffin. Sections of $3.5 \mu \mathrm{m}$ thickness were rehydrated in a descending series of ethanolic solutions and deparaffinized in xylene. In staining with KDM4A, KDM4B and KDM4D the samples were pre-treated in a microwave oven in Tris-EDTA buffer in $\mathrm{pH} 9$ for $15 \mathrm{~min}$ for KDM4A and KDM4D and 10 min for KDM4B. For SIRT1-4, the samples were also pre-treated in a microwave owen but in Tris-EDTA buffer in $\mathrm{pH}$ 6 for $17 \mathrm{~min}$ for SIRT1 and SIRT4 and $12 \mathrm{~min}$ for SIRT2 and SIRT3. Next, for KDM4A, KDM4B and KDM4D staining, sections were incubated in a $3 \% \mathrm{H}_{2} \mathrm{O}_{2}$ solution for 15 min to block the endogenous peroxidase activity. In SIRT1-4 stainings $3 \% \mathrm{H}_{2} \mathrm{O}_{2}$ was replaced with Dako REAL ${ }^{\mathrm{TM}}$ Peroxidase-Blocking solution (Dako S2023, Dako Denmark A/S, Glostrup, Denmark). Samples were incubated with primary antibodies (Table I) at $+4 \mathrm{C}$ temperature overnight (Table I). After the slides were incubated with secondary biotinylated antibody, the immunostaining was carried out with VECTASTAIN ${ }^{\circledR}$ Elite ABC KIT (Vector Laboratories, Inc., Burlingame, CA, USA) for SIRT1 and SIRT 4, Lab Vision ${ }^{\text {TM }}$ UltraVision ${ }^{\text {TM }}$ Large Volume Detection System (Thermo Fisher Scientific, Fremont, USA) for SIRT 2 and Dako REAL ${ }^{\mathrm{TM}}$ EnVision $^{\mathrm{TM}}$ Detection System (Dako Denmark A/S, Glostrup, Denmark) for SIRT 3, KDM41, KDM4B 

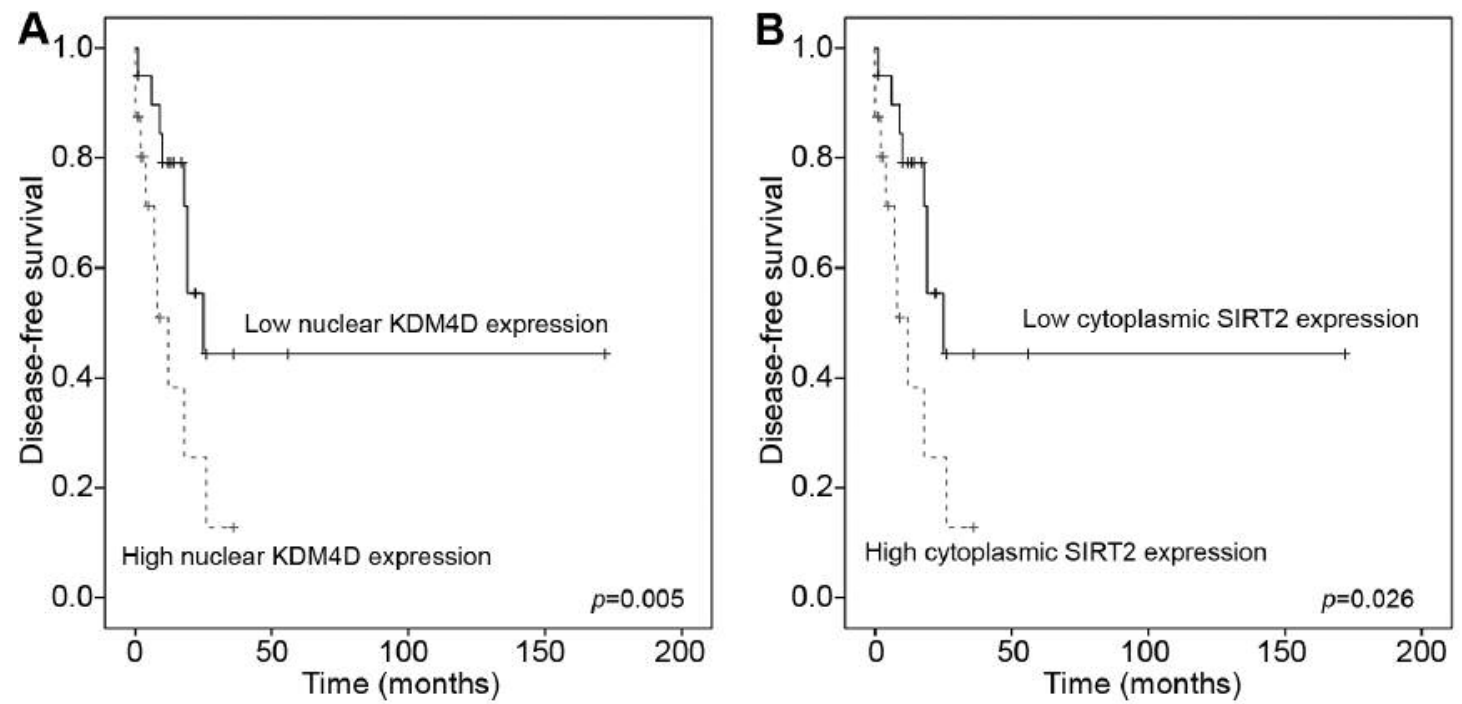

Figure 1. Kaplan-Meier curves showing disease-free survival (DFS) according to nuclear lysine (K)-specific demethylase $4 D(K D M 4 D)$ expression (A) and cytoplasmic Sirtuin 2 (SIRT2) expression (B) in benign pancreatic tissue of resection margin .

Table I. Immunohistochemical methods.

\begin{tabular}{|c|c|c|c|}
\hline Primary antibody & Manufacturer of the primary antibody & Dilution & Immunostaining method \\
\hline $\begin{array}{l}\text { KDM4A } \\
(\text { ab104831) }\end{array}$ & Abcam, Cambridge, UK & $1: 2,000$ & $\begin{array}{l}\text { Dako REAL }{ }^{\mathrm{TM}} \text { EnVision }{ }^{\mathrm{TM}} \text { Detection System } \\
\text { (Dako Denmark A/S, Glostrup, Denmark) }\end{array}$ \\
\hline $\begin{array}{l}\text { KDM4B } \\
\text { (ab103129) }\end{array}$ & Abcam, Cambridge, UK & $1: 250$ & $\begin{array}{l}\text { Dako REAL }{ }^{\mathrm{TM}} \text { EnVision }{ }^{\mathrm{TM}} \text { Detection System } \\
\text { (Dako Denmark A/S, Glostrup, Denmark) }\end{array}$ \\
\hline $\begin{array}{l}\text { KDM4D } \\
(\text { ab93694) }\end{array}$ & Abcam, Cambridge, UK & $1: 1,500$ & $\begin{array}{l}\text { Dako REAL }{ }^{\mathrm{TM}} \text { EnVision }{ }^{\mathrm{TM}} \text { Detection System } \\
\text { (Dako Denmark A/S, Glostrup, Denmark) }\end{array}$ \\
\hline $\begin{array}{l}\text { SIRT1 } \\
(\text { ab166821) }\end{array}$ & Abcam, Cambridge, UK & $1: 200$ & $\begin{array}{l}\text { VECTASTAIN }{ }^{\circledR} \text { Elite ABC KIT } \\
\text { (Vector Laboratories, Inc., Burlingame, USA) }\end{array}$ \\
\hline $\begin{array}{l}\text { SIRT2 } \\
\text { (NBP1-50461) }\end{array}$ & Novus Biologicals, Littleton, USA & $1: 1,500$ & $\begin{array}{l}\text { Lab Vision }^{\mathrm{TM}} \text { UltraVision }^{\mathrm{TM}} \text { Large Volume Detection } \\
\text { System (Thermo Fisher Scientific, Fremont, USA) }\end{array}$ \\
\hline $\begin{array}{l}\text { SIRT3 } \\
\text { (sc-99143) }\end{array}$ & Santa Cruz Biotechnology Inc., Dallas, USA & $1: 250$ & $\begin{array}{l}\text { Dako REAL }{ }^{\mathrm{TM}} \text { EnVision }{ }^{\mathrm{TM}} \text { Detection System } \\
\text { (Dako Denmark A/S, Glostrup, Denmark) }\end{array}$ \\
\hline $\begin{array}{l}\text { SIRT4 } \\
\text { (ab 10140) }\end{array}$ & Abcam, Cambridge, UK & $1: 100$ & $\begin{array}{c}\text { VECTASTAIN }{ }^{\circledR} \text { Elite ABC KIT } \\
\text { (Vector Laboratories, Inc., Burlingame, USA) }\end{array}$ \\
\hline
\end{tabular}

KDM4: Lysine (K) - specific demethylase; SIRT: sirtuin.

and KDM4D according to the manufacturer's instructions. Between the stages of the immunostaining procedure, the slides were washed with Tris-buffered saline (TBS). 3,3-Diamaminobenzidine was used as a chromogen and the slides were counterstained with Mayer's haematoxylin and finally mounted.

Statistical analyses. For statistical analyses, the intensity (0-3) was multiplied by the percentage of stained cells to all malignant cells ( 0 $100 \%$ ) resulting in a continuous variable of 0-300. Both the intensity and the extent of immunostaining were separately evaluated in nuclei and cytoplasm, and separately in adenocarcinoma cells and in cells of exocrine pancreas at the resection margins. Mann-Whitney test and Spearman's rho test were used to determine the significance of the results with the exception of survival analyses where the continuous variable was divided into two classes (low or high expression) based on the median expression of each variable.

Grade was divided into well-to-moderate differentiation or poor differentiation and T-class was handled in statistical analyses as T1-2 or T3-4. Associations between KDM4 and SIRT expressions and patient survival were analysed with the Kaplan-Meier method, and the statistical significance of the differences was evaluated using the logrank test. Disease-free survival (DFS) was calculated from the date of diagnosis to the date of the first confirmed relapse, either local or distant. Overall survival (OS) was calculated from the date of diagnosis to the time of death from any reason. Cox regression analysis was applied in multivariate analysis. Statistical analyses were carried out by using IBM SPSS Statistics 22.0.0.0 software (SPSS, Chicago, IL, USA) and the results were considered significant if the two-sided $p$-value was $<0.05$. 


\section{Results}

Staining patterns in malignant tissue. KDM4A was detected in all but one of the cases $(n=78)$ both in nuclei and cytoplasm (Figure 1, Table II). Nuclear staining intensity ranged from weak (+) to strong (+++) and majority of the cases showed $50 \%$ or more extent of immunostaining. Cytoplasmic staining intensity varied from weak $(+)$ to strong $(+++)$, but only three samples showed strong immunopositivity. Extent of cytoplasmic immunostaining was $100 \%$ in all cases and in nucleus it ranged from 1\%-100\%. Cytoplasmic KDM4B and KDM4D expression was identified in 78 cases and in these cases all cells had at least some immunopositivity. Cytoplasmic intensity varied in both stainings from weak $(+)$ to moderate $(++)$. Only one sample showed strong $(+++)$ cytoplasmic KDM4B expression. Weak KDM4B nuclear positivity was seen in 50 cases. Nuclear KDM4D expression was detected in 26 samples where three of them showed strong (+++) staining. The extent of nuclear staining varied from $5 \%$ to $100 \%$ in these stainings. Membrane-associated KDM4A was observed in 13 and KDM4D in 21 cases. Two samples were not evaluable because of the exhaustion of the blocks or the occurrence of non-representative areas.

Majority of samples showed cytoplasmic SIRT1-4 expression and the intensity of expression varied from weak (+) to strong $(+++)$ with all antibodies. The magnitude of cytoplasmic expression was $100 \%$ in stainings SIRT1 and SIRT4. In stainings with SIRT2-3, the magnitude of cytoplasmic expression varied from $20-100 \%$. The majority of the nuclei were negative with regards to SIRT1, SIRT3 and SIRT4 in malignant cells. 14 cases $(14.6 \%)$ showed weak $(+)$ or moderate $(++)$ nuclear SIRT2 immunopositivity and the percentage of positive cells ranged from 5-30\% in the malignant cells. 27 cases showed membraneassociated SIRT4 expression and tumour microenvironmental stromal SIRT2 staining was observed in majority of the samples $(n=92)$. Three of the samples were not evaluable.

Staining patterns in benign tissue from resection margins. KDM4A expression was detected both in nuclei and cytoplasm in majority of the cases $(97.6 \%)$ and intensity varied mostly from weak (+) to moderate $(++)$. Two of the cases showed strong nuclear staining. The extent of nuclear staining ranged from $1-100 \%$, but cytoplasmic staining was observed in all cells in most samples. KDM4B expression was observed in 32 of the cases in cytoplasm and in 23 cases in nuclei. Only three samples were completely negative. Staining intensity was mainly weak, both in nuclei and cytoplasm. In cytoplasm, the extent of staining was $100 \%$ and in nuclei the magnitude of immunostaining was mostly $50 \%$ or less.

All of the samples except one showed cytoplasmic KDM4D staining $(n=42)$, but 18 cases showed also KDM4D nuclear staining. Intensity varied mainly from weak to moderate both in cytoplasm and nuclei, while two cases showed strong nuclear KDM4D staining. The extent of cytoplasmic staining was $100 \%$ in all samples with cytoplasmic immunopositivity, but the magnitude of nuclear staining varied between 10-100\%. Overall, 38 cases were not evaluable due to exhaustion of the blocks or the occurrence of non-representative areas.

Cytoplasmic SIRT1 expression was recorded in all except two of the cases $(n=30)$. Intensity varied from weak to strong. Also, SIRT3 staining was detected in all except two of the cases $(n=32)$ and the intensity was mainly weak or moderate in cytoplasm but 3 cases showed strong cytoplasmic SIRT3 immunoreaction. Weak or moderate cytoplasmic SIRT2 expression was detected in all cases $(n=37)$ and SIRT4 expression in all except four of the cases $(n=29)$. The extent of SIRT1 and SIRT4 immunostainings were $100 \%$ in those samples, where the extent of immunoreactivity varied from $20 \%$ to $100 \%$ with SIRT2-3 immunostainings in benign tissue areas. Nuclei were completely negative in all SIRT stainings except for two samples that showed weak nuclear SIRT1 and SIRT4 expression. Depending on a staining, 60 to 70 samples were not evaluable due to exhaustion of the blocks or the occurrence of non-representative benign tissue.

Association with clinical parameters. KDM4A expression in the nuclei of adenocarcinoma cells had an inverse correlation with the primary tumor size as millimetres $(p=0.002$; correlation coefficient -0.474$)$. In benign pancreas in resection margins, nuclear KDM4A correlated inversely with the number of metastatic lymph nodes $(p=0.03$; correlation coefficient -0.452). We did not find any associations between KDM4B expression and studied parameters.

High nuclear KDM4D expression in benign pancreatic cells from resection margins associated with T-class T1-2 compared to T3-4 ( $p=0.005)$. Cytoplasmic KDM4D in adenocarcinoma cells was overexpressed in grade I-II tumors, compared to poorly differentiated carcinomas $(p=0.043)$.

High cytoplasmic SIRT1 immunostaining in benign pancreatic tissue from resection margins associated with poor differentiation $(p=0.033)$. In contrast, high cytoplasmic SIRT3 expression in benign pancreatic tissue was connected with lower grade $(p=0.034)$. SIRT4 did not show any associations with the studied parameters.

Survival analysis. High nuclear KDM4D expression in benign pancreatic tissue of resection margins associated with significantly shorter DFS ( $p=0.005$ ) (Figure 2$)$. In multivariate analysis this was still the most significant predictor of DFS $(\mathrm{OR}=8.00 ; 95 \% \mathrm{CI}=1.87-33.9 ; p=0.005)$ when the number of metastatic lymph nodes $(\mathrm{OR}=1.59 ; 95 \% \mathrm{CI}=1.10-2.31$; $p=0.015)$ and $\mathrm{T}$-class $(\mathrm{OR}=2.57 ; 95 \% \mathrm{CI}=0.543-12.2 ; p=0.23)$ were also included in the model. 
Table II. Percentage of evaluable cases showing any expression of KDM4A, KDM4B, KDM4D and SIRT1-4.

\begin{tabular}{lccccc}
\hline & \multicolumn{2}{c}{ Adenocarcinoma cells } & & \multicolumn{2}{c}{ Benign pancreatic tissue } \\
\cline { 2 - 3 } \cline { 6 - 6 } & Nuclear & Cytoplasm & & Nuclear & Cytoplasm \\
\hline KDM4A & 98.7 & 97.5 & & 100.0 & 97.6 \\
KDM4B & 63.3 & 98.7 & & 65.7 & 91.4 \\
KDM4D & 33.3 & 100.0 & & 41.9 & 97.7 \\
SIRT1 & 0.1 & 100.0 & & 3.1 & 93.8 \\
SIRT2 & 14.6 & 93.8 & & 0.0 & 100.0 \\
SIRT3 & 1.0 & 98.0 & & 0.0 & 94.1 \\
SIRT4 & 0.0 & 100.0 & & 3.0 & 87.9 \\
\hline
\end{tabular}

High cytoplasmic SIRT2 expression in benign pancreatic tissue of resection margins associated with shorter DFS in univariate analysis $(p=0.026)$. However, in multivariate analysis this association was not significant $(\mathrm{OR}=2.05$; $95 \% \mathrm{CI}=0.49-8.52 ; p=0.32)$ when the number of metastatic lymph nodes $(\mathrm{OR}=1.94 ; 95 \% \mathrm{CI}=0.90-4.18 ; p=0.091)$ and T-class $(\mathrm{OR}=1.29 ; 95 \% \mathrm{CI}=0.25-6.64 ; p=0.76)$ were included to the model. No associations with OS were found.

\section{Discussion}

Based on these results, expression of KDM4A-D or SIRT14 , did not influence the prognosis of patients with pancreatic adenocarcinoma, when the expression is assessed in malignant cells. On the other hand, nuclear KDM4D expression in benign exocrine pancreatic cells from resection margins appeared as a prognostic factor in surgically treated pancreatic adenocarcinomas exceeding the prognostic value of $\mathrm{T}$ and $\mathrm{N}$ of the TNM classification. A similar tendency was observed with a high cytoplasmic SIRT2 expression.

A recent comprehensive integrated genomic analysis of pancreatic adenocarcinomas revealed KDM6A as a new candidate driver of pancreatic carcinogenesis (24). According to our knowledge, this is the first study to assess KDM4 expression in pancreatic cancer with clinical material. The preclinical evidence is also limited but has suggested that KDM4B can epigenetically up-regulate transcription factor Zeb1 expression and consequently accelerate epithelialmesenchymal transition (25). KDM4 isoenzyme overexpression in malignant compared to benign tissues has been reported in breast, endometrial, lung and prostate cancers (26-29). In our recent work with Hodgkin lymphomas, strong KDM4D expression in Reed-Sternberg cells associated with poorer relapse-free survival in the limited-stage patients, but only in those who had received involved-field radiotherapy (14).

Previously, nuclear KDM4D expression has been shown to be a negative prognostic factor in lung carcinoma and especially in squamous cell carcinoma (30). Compared to other KDM4 isoenzymes, KDM4D stimulates proliferation and cell survival in vitro in a p53 mediated manner and has also a vital role in DNA double-strand break repair in a PARP1-dependent manner $(13,31)$. While expression in malignant pancreatic cells had no prognostic significance, high nuclear KDM4D expression in benign pancreatic tissue taken from resection margins predicted an earlier recurrence. Notably, only $32 \%$ of patients with low nuclear KDM4D expression relapsed during the follow-up. Patients with high nuclear KDM4D expression had an 8-fold risk of early relapse when T-class and the number of lymph node metastases were included to the multivariate analysis. Nuclear KDM4D expression assessed from resection margins of the benign pancreas may thus be used as a marker of early recurrence of the tumor. It is possible that tumor cells stimulate adjacent normal exocrine cells to increase KDM4D and change their methylation status. However, this hypothesis is partly contradicted by the fact that nuclear KDM4D in benign pancreatic tissue associated inversely with T-class. Another possibility is that inflammatory cells within the tumor tissue influence KDM4D expression in benign cells and KDM4D expression in benign cells of the exocrine pancreas.

Nuclear KDM4A expression in cancer cells associated with smaller tumor size. This is in contrast with previous reports in other tumor types such as breast, colon and non-small cell lung carcinomas, where KDM4A has been suggested to have tumor-promoting effects $(9,32)$. Nevertheless, KDM4A has not, according to our knowledge, been assessed previously in pancreatic cancer and thus it seems that KDM4A may have cancer-specific roles. The expression in benign pancreatic cells in resection margins correlated inversely with the number of metastatic lymph nodes suggesting opposing roles compared to KDM4D even though association with recurrence was not observed with KDM4A.

While KDM4 expression has been poorly characterized in pancreatic cancer, sirtuins have been studied considerably more, but with somewhat conflicting results. In general, SIRT1 has been described as either tumour suppressor or tumour promoter, depending on cellular context or the effect on a specific signalling pathway (33). SIRT1-mediated deacetylation may lead, in vitro, to the attenuated function of several tumour suppressors, including p53, p73, and HIC1 $(17,34)$. In pancreatic cancer SIRT1 overexpression substantially attenuates the effect of gemcitabine and associates with poor differentiation and poor survival (16, 35). Decrease of nuclear and increase of cytoplasmic SIRT1 has been demonstrated in vitro to stimulate pancreatic cancer precursor, acinar-to-ductal metaplasia, while SIRT1 inhibition is potent to diminish the viability of pancreatic cancer cells (36). We, however, did not find any association of SIRT1 expression in malignant cells with clinical parameters. In breast cancer, SIRT2 associates with shorter 

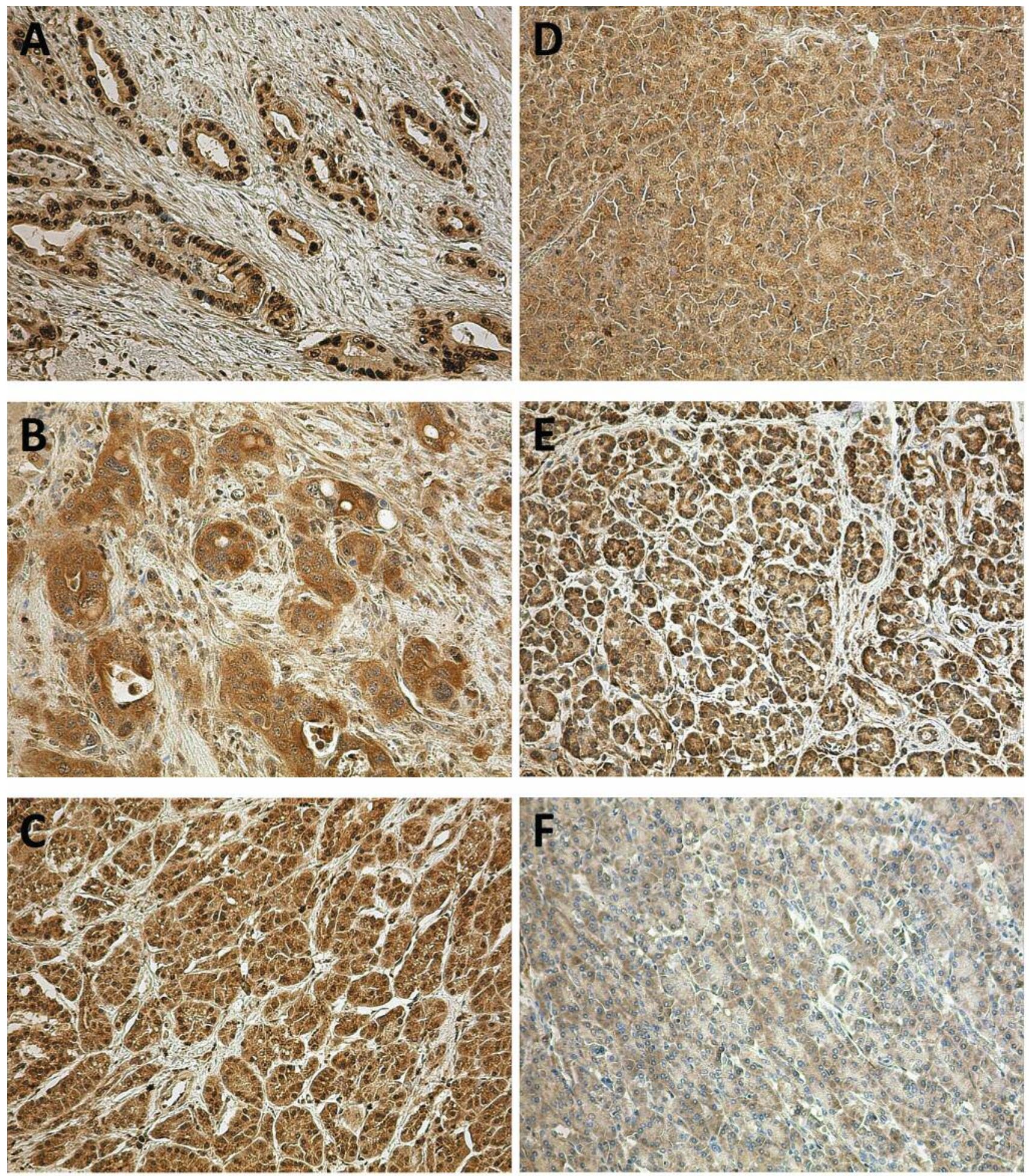

Figure 2. Strong (A) and weak (B) nuclear KDM4A expression in malignant pancreatic tissue. Strong $(C)$ and weak (D) nuclear KDM4D expression in benign pancreatic tissue of the resection margin. Strong $(E)$ and weak $(F)$ cytoplasmic SIRT2 expression in benign pancreatic tissue of the resection margin (Low magnification $\times 10)$. 
recurrence-free survival and DFS, but only in grade 2-3 tumours (23). We also performed survival analysis separately in tumours with different grade, but maybe due to limited sample size this did not reveal any novel significances (data not shown). The clinicopathological value of SIRT3 and SIRT4 seems more limited in pancreatic cancer, in contrast to previous studies that suggested tumour suppressive effects of SIRT3 in tissue microarray sections of pancreatic adenocarcinomas (37). According to our knowledge, SIRT4 expression has not been previously described in pancreatic neoplasias.

In samples from resection margins, cytoplasmic SIRT1 expression in benign pancreatic cells associated with poor differentiation in pancreatic cancer. Analogously, cytoplasmic SIRT2 expression in benign cells in resection margins was linked with recurrence, although it had no independent prognostic value in multivariate analysis. These results, as with KDM4D, suggest that benign cells undergo epigenetic changes in view of histone modifications which are reflected in parameters of tumor behaviour. In case of SIRT1 and 2, the associations were linked to cytoplasmic positivity implying an increased synthesis in the benign cells. As far as lymphocyte activation is concerned in experimental studies, SIRT1 has been shown to activate T cells (38). On the other hand, SIRT2 has been found to influence macrophage polarisation and protect from experimental colitis (39). Such experimental results might partly be due to tumor induced immunity.

Our results suggest that histone modifications reflected by changes in KDM and SIRT1-4 enzymes do not play a significant role in pancreatic adenocarcinoma cells. A different type of expression was, however seen in nuclear KDM4D and cytoplasmic SIRT2 expression between tumor cells and benign exocrine cells. Interestingly, high nuclear KDM4D expression from benign cells of surgical resection margins predicted an earlier recurrence and a high cytoplasmic SIRT2 expression showed a similar tendency. These observations indicate that benign exocrine pancreatic cells undergo changes in histone methylation in relation to a malignant disease in adjacent pancreas. Whether this is due to a local inflammation, some other stromal action or direct paracrine effects of tumor cells remains to be clarified in future studies.

\section{Conflicts of Interest}

The Authors declare that they have no conflict of interest.

\section{Acknowledgements}

The Authors would like to thank Manu Tuovinen and Riitta Vuento for their technical expertise in preparation of the immunohistochemical stainings. This work was supported by grants from Mary and Georg C. Ehrnrooth Foundation and Ida Montin Foundation.

\section{References}

1 Rahib L, Smith BD, Aizenberg R, Rosenzweig AB, Fleshman $\mathrm{JM}$ and Matrisian LM: Projecting cancer incidence and deaths to 2030: The unexpected burden of thyroid, liver, and pancreas cancers in the united states. Cancer Res 74: 2913-2921, 2014.

2 Ryan DP, Hong TS and Bardeesy N: Pancreatic adenocarcinoma. N Engl J Med 371: 1039, 2014.

3 Bailey P, Chang DK, Nones K, Johns AL, Patch AM, Gingras MC, Miller DK, Christ AN, Bruxner TJC, JC, Quinn MC, Nourse C, Murtaugh LC, Harliwong I, Idrisoglu S, Manning S, Nourbakhsh E, Wani S, Fink L, Holmes O, Chin V, Anderson MJ, Kazakoff S, Leonard C, Newell F, Waddell N, Wood S, Xu Q, Wilson PJ, Cloonan N, Kassahn KS, Taylor D, Quek, K, Robertson A, Pantano L, Mincarelli L. Sanchez LN, Evers L. Wu J, Pinese M, Cowley MJ, Jones MD, Colvin EK, Nagrial AM, Humphrey ES, Chantrill LA, Mawson A, Humphris J, Chou A, Pajic M, Scarlett CJ, Pinho AV, Giry-Laterriere M, Rooman I, Samra JS, Kench JG, Lovell JA, Merrett ND, Toon CW, Epari K, Nguyen NQ, Barbour A, Zeps N, Moran-Jones K. Jamieson NB, Graham JS. Duthie F, Oien K, Hair J, Grützmann R, Maitra A, Iacobuzio-Donahue CA, Wolfgang CL, Morgan RA, Lawlor RT, Corbo V, Bassi C, Rusev B, Capelli P, Salvia R, Tortora G, Mukhopadhyay D, Petersen GM, Munzy DM, Fisher WE, Karim SA, Eshleman JR, Hruban RH, Pilarsky, C, Morton JP, Sansom OJ, Scarpa A, Musgrove EA, Hofmann O, Sutherland RL, Wheeler DA, Gill AJ, Gibbs RA, Pearson JV, Waddell N, Biankin AV and Grimmond SM: Genomic analyses identify molecular subtypes of pancreatic cancer. Nature 531: 47, 2016.

4 Ducreux M, Cuhna AS, Caramella C, Hollebecque A, Burtin P, Goéré D, Seufferlein T, Haustermans K, Van Laethem JL, Conroy $\mathrm{T}$ and Arnold D: ESMO Guidelines Committee. Cancer of the pancreas: ESMO Clinical Practice Guidelines for diagnosis, treatment and follow-up. Ann Oncol 26: 56-68, 2015.

5 Rossi E, Bagalà C, Inzani F, Leoncini E, Brunelli C, Lanza P, Basso M, Mattiucci GC, Cassano A, Rindi G, Barone C and Schinzari G: RUNX3 as a potential predictor of metastasis in human pancreatic cancer. In Vivo 31: 833-840, 2017.

6 Ploquin A, Truant S, Piessen G, Vuagnat P, Baldini C, Cattan S and Hebbar M: Locally advanced or metastatic pancreatic adenocarcinoma: Easily available factors of predictive prolonged survival under gemcitabine. In Vivo 31: 731-735, 2017.

7 Ikemoto T, Shimada M, Ishikawa D, Kawashita Y, Teraoku H, Yoshikawa M, Yamada S, Saito YU, Morine Y and Imura S: Peripheral Tr1 and Foxp3 Treg as markers of recurrent malignancies in patients with hepato-biliary pancreatic cancers. Anticancer Res 37: 5541-5552, 2017.

8 Lomberk GA, Iovanna J and Urrutia R: The promise of epigenomic therapeutics in pancreatic cancer. Epigenomics 8: 831-842, 2016.

9 Soini Y, Kosma V and Pirinen R: KDM4A, KDM4B and KDM4C in non-small cell lung cancer. Int $\mathrm{J}$ Clin Experiment Pathol 8: 12922, 2015.

10 Liang G, Egger G, Jones PA and Aparicio A: Epigenetics in human disease and prospects for epigenetic therapy. Nature 429: 457-463, 2004.

11 Kanwal R, Gupta K and Gupta S: Cancer epigenetics: An introduction. Methods Mol Biol 1238: 3-25, 2015.

12 Sharma S, Kelly TK and Jones PA: Epigenetics in cancer. Carcinogenesis 31: 27-36, 2010. 
13 Kim T, Oh S, Shin S and Janknecht R: Regulation of tumor suppressor p53 and HCT116 cell physiology by histone demethylase JMJD2D/KDM4D. PloS one 7: e34618, 2012.

14 Bur H, Haapasaari K, Turpeenniemi-Hujanen T, Kuittinen O, Auvinen P, Marin K, Soini Y and Karihtala P: Strong KDM4B and KDM4D expression associates with radioresistance and aggressive phenotype in classical Hodgkin lymphoma. Anticancer Res 36: 4677-4683, 2016.

15 Kawamura A, Münzel M, Kojima, Yapp C, Bhushan B, Goto Y, Tumber A, Katoh T, King ON, Passioura T, Walport LJ, Hatch SB, Madden S, Muller S, Brennan PE, Chowdhury R, Hopkins RJ, Suga H and Schofield CJ: Highly selective inhibition of histone demethylases by de novo macrocyclic peptides. Nat Commun 8: 14773, 2017.

16 Stenzinger A, Endris V, Klauschen F, Sinn B, Lorenz K, Warth A, Goeppert B, Ehemann V, Muckenhuber A, Kamphues C, Bhra $M$, Neuhaus $P$ and Weichert W: High SIRT1 expression is a negative prognosticator in pancreatic ductal adenocarcinoma. BMC Cancer 13: 450, 2013.

17 Zhenghong L and Deyu F: The roles of SIRT1 in cancer. Genes Cancer 4: 97-103, 2013.

18 Choi J and Mostoslavsky R: Sirtuins, metabolism, and DNA repair. Curr Opin Genet Dev 26: 24, 2014.

19 Jing $\mathrm{H}$ and Lin H: Sirtuins in epigenetic regulation. Chem Rev 115: 2350, 2015.

20 Yuan H, Su L and Chen WY: The emerging and diverse roles of sirtuins in cancer: A clinical perspective. Onco Targets Ther 6: 1399-1416, 2013.

21 Kaufmann T, Kukolj E, Brachner A, Beltzung E, Bruno M, Kostrhon S, Opravil S, Hudecz O, Mechtler K, Warren G and Slade D: SIRT2 regulates nuclear envelope reassembly through ANKLE2 deacetylation. J Cell Sci 129: 4607-4621, 2016.

22 Bosch-Presegué L and Vaquero A: Sirtuin-dependent epigenetic regulation in the maintenance of genome integrity. FEBS J 282: 1745-1767, 2015.

23 McGlynn LM, Zino S, MacDonald AI, Curle J, Reilly JE, Mohammed ZM, McMillan DC, Mallon E, Payne AP, Edwards J and Shiels PG: SIRT2: Tumour suppressor or tumour promoter in operable breast cancer? Eur J Cancer 50: 290-301, 2014.

24 Waddell N, Pajic M, Patch AM, Chang DK, Kassahn KS, Bailey P, Johns AL, Miller D, Nones K, Quek K, Quinn MC, Robertson AJ, Fadlullah MZ, Bruxner TJ, Christ AN, Harliwong I, Idrisoglu S, Manning S, Nourse C, Nourbakhsh E, Wani S, Wilson PJ, Markham E, Cloonan N, Anderson MJ, Fink JL, Holmes O, Kazakoff SH, Leonard C, Newell F, Poudel B, Song S, Taylor D, Waddell N, Wood S, Xu Q, Wu J, Pinese M, Cowley MJ, Lee HC, Jones MD, Nagrial AM, Humphris J, Chantrill LA, Chin V, Steinmann AM, Mawson A, Humphrey ES, Colvin EK, Chou A, Scarlett CJ, Pinho AV, Giry-Laterriere M, Rooman I, Samra JS, Kench JG, Pettitt JA, Merrett ND, Toon C, Epari K, Nguyen NQ, Barbour A, Zeps N, Jamieson NB, Graham JS, Niclou SP, Bjerkvig R, Grützmann R, Aust D, Hruban RH, Maitra A, IacobuzioDonahue CA, Wolfgang CL, Morgan RA, Lawlor RT, Corbo V, Bassi C, Falconi M, Zamboni G, Tortora G, Tempero MA; Australian Pancreatic Cancer Genome Initiative, Gill AJ, Eshleman JR, Pilarsky C, Scarpa A, Musgrove EA, Pearson JV, Biankin AV and Grimmond SM: Whole genomes redefine the mutational landscape of pancreatic cancer. Nature 518: 495-501, 2015.

25 Linlin SL, Wang WQ, Li Y and Wang X: KDM4B promotes epithelial-mesenchymal transition through up-regulation of
ZEB1 in pancreatic cancer. Acta Biochim Biophys Sin (Shanghai) 47: 997-1004, 2015.

26 Berry WL and Janknecht R: KDM4/JMJD2 histone demethylases: Epigenetic regulators in cancer cells. Cancer Res 73: 2936-2942, 2013.

27. Qiu MT, Fan Q, Zhu Z, Kwan SY, Chen L, Chen JH, Ying ZL, Zhou Y, Gu W, Wang LH, Cheng WW, Zeng J, Wan XP, Mok $\mathrm{SC}$, Wong KK and Bao W: KDM4B and KDM4A promote endometrial cancer progression by regulating androgen receptor, c-myc, and p27kip1. Oncotarget 6: 31702, 2015.

28 Mallette $\mathrm{F}$ and Richard S: JMJD2A promotes cellular transformation by blocking cellular senescence through transcriptional repression of the tumor suppressor CHD5. Cell Reports 2: 1233-1243, 2012.

29 Cloos PA, Christensen J, Agger K, Maiolica J, Antal T, Hansen KH and Helin $\mathrm{K}$ : The putative oncogene GASC1 demethylates tri- and dimethylated lysine 9 on histone H3. Nature 442: 307-311, 2006.

30 Uimonen K, Merikallio H, Pääkkö P, Harju T, Mannermaa A, Palvimo J, Kosma VM and Soini Y: GASC1 expression in lung carcinoma is associated with smoking and prognosis of squamous cell carcinoma. Histol Histopathol 29: 797, 2014.

31 Khoury-Haddad H, Nadar-Ponniah PT, Awwad S and Ayoub N: The emerging role of lysine demethylases in DNA damage response: Dissecting the recruitment mode of KDM4D/JMJD2D to DNA damage sites. Cell Cycle 14: 950-958, 2015.

32 Guerra-Calderas L, González-Barrios R, Herrera LA, Cantú de León D and Soto-Reyes E: The role of the histone demethylase KDM4A in cancer. Cancer Genet 208: 215-224, 2015.

33 Kleszcz R, Paluszczak J and Baer-Dubowska W: Targeting aberrant cancer metabolism - the role of sirtuins. Pharmacol Rep 67: 1068-1080, 2015.

34 Vaziri H, Dessain SK, Ng Eaton E, Imai SI, Frye RA, Pandita TK, Guarente L and Weinberg RA: p53 Deacetylase. hSIR2SIRT1 functions as an NAD-dependent p53 deacetylase. Cell 107: 149-159, 2001.

35 Zhang JG, Hong DF, Zhang CW, Sun XD, Wang ZF, Shi Y, Liu JW, Shen GL, Zhang YB, Cheng J, Wang CY and Zhao G: Sirtuin 1 facilitates chemoresistance of pancreatic cancer cells by regulating adaptive response to chemotherapy-induced stress. Cancer Sci 105: 445-454, 2014.

36 Wauters E, Sanchez-Arévalo Lobo VJ, Pinho AV, Mawson A, Herranz D, Wu J, Cowley MJ, Colvin EK, Njicop EN, Sutherland RL, Liu T, Serrano M, Bouwens L, Real FX, Biankin AV and Rooman I: Sirtuin-1 regulates acinar-to-ductal metaplasia and supports cancer cell viability in pancreatic cancer. Cancer Res 73: 2357, 2013.

37 McGlynn LM, McCluney S, Jamieson NB, Thomson J, MacDonald AI, Oien K, Dickson EJ, Carter CR, McKay CJ and Shiels PG: SIRT3 \& SIRT7: Potential novel biomarkers for determining outcome in pancreatic cancer patients. PloS one 10: e0131344, 2015.

38 Zou T, Yang Y, Xia F, Huang A, Gao X, Fang D, Xiong S and Zhang J: Resveratrol inhibits $\mathrm{CD} 4^{+} \mathrm{T}$ cell activation by enhancing the expression and activity of Sirt1. PloS one 8: e75139, 2013.

39 Lo Sasso G, Menzies KJ, Mottis A, Piersigilli A, Perino A, Yamamoto H, Schoonjans K and Auwerx J: SIRT2 deficiency modulates macrophage polarization and susceptibility to experimental colitis. PloS one 9: e103573, 2014.

Received January 4, 2018

Revised February 14, 2018 Accepted February 15, 2018 